\title{
LINK OF GROUPS AND HOMOGENEOUS HÖRMANDER OPERATORS
}

\author{
ALESSIA ELISABETTA KOGOJ AND ERMANNO LANCONELLI
}

(Communicated by David S. Tartakoff)

\begin{abstract}
We study a notion of link of Lie groups suggested by the structure of the partial differential operators of Kolmogorov type. As an application of our link procedure we construct explicit examples of stratified Lie groups, with dimension and step arbitrarily large. We also give a set of examples of hypoelliptic second-order operators which are left translation invariant and homogeneous of degree two on the previous groups.
\end{abstract}

\section{INTRODUCTION}

In this paper we study the notion of link of homogeneous groups first explicitly fixed in 9. This notion seems not completely new and it is somehow related to the notion of fiber product. However, our presentation, which is inspired by the structure of the operators introduced by Kolmogorov in studying diffusion processes from a probabilistic point of view (see [12, 11]) seems more suitable to be applied to the theory of second-order partial differential operators of Hörmander type.

In order to clarify our main purpose, we first recall that the above-mentioned Kolmogorov operator is the following one:

$$
\mathcal{K}=\Delta_{x}+x \nabla_{y}-\partial_{t}
$$

where $x, y \in \mathbb{R}^{n}$ and $t \in \mathbb{R} . \Delta_{x}$ and $\nabla_{y}$ respectively denote the classical Laplace operator and the Euclidean gradient in $\mathbb{R}^{n}$. Moreover, $x \nabla_{y}$ stands for the first-order differential operator $\sum_{j=1}^{n} x_{j} \partial_{y_{j}}$.

It has been proved in 12 that $\mathcal{K}$ is left translation invariant on the Lie group $\left(\mathbb{R}^{2 n+1}, \circ\right)$ whose composition law is given by

$$
(x, y, t) \circ\left(x^{\prime}, y^{\prime}, t^{\prime}\right):=\left(x+x^{\prime}, y+y^{\prime}+t^{\prime} x, t+t^{\prime}\right) .
$$

The dilations

$$
\delta_{\lambda}: \mathbb{R}^{2 n+1} \longrightarrow \mathbb{R}^{2 n+1}, \quad \delta_{\lambda}(x, y, t)=\left(\lambda x, \lambda^{3} y, \lambda^{2} t\right), \quad \lambda>0,
$$

are homomorphisms of $\left(\mathbb{R}^{2 n+1}, \circ\right)$, so that

$$
\mathbb{K}=\left(\mathbb{R}^{2 n+1}, \circ, \delta_{\lambda}\right)
$$

Received by the editors April 14, 2005 and, in revised form, January 15, 2006.

2000 Mathematics Subject Classification. Primary 35K70, 35H10; Secondary 43A80, 35H20.

Key words and phrases. Hörmander operators, Kolmogorov operators, partial differential operators on homogeneous Lie groups. 
is a homogeneous Lie group. It easy to verify that $\mathcal{K}$ also is $\delta_{\lambda}$-homogeneous of degree two.

We shall say that $\mathbb{K}$ is the Kolmogorov group related to the first-order partial differential operator

$$
Y:=x \nabla_{y}-\partial_{t} .
$$

It seems quite natural to expect that replacing $\Delta_{x}$ in (11) with a suitable subLaplacian on a Carnot group, we should obtain a new operator with an underlying structure of a homogeneous Lie group.

Actually, as an application of our general results, we shall see that if $\Delta_{\mathbb{G}}$ is any sub-Laplacian and $Y$ is a first-order partial differential operator which is transverse to $\mathbb{G}$ (in the sense of Definition 4.5), then

$$
\mathcal{L}=\Delta_{\mathbb{G}}+Y
$$

is left translation invariant and homogeneous of degree two on a new homogeneous group obtained by linking $\mathbb{G}$ with the Kolmogorov group related to $Y$.

As another application of our linking procedure, we are able to construct explicit examples of homogeneous and Carnot groups of dimension and step arbitrarily large. We are also able to give new explicit examples of degenerate elliptic and ultraparabolic operators of Hörmander type.

The study of this class of operators, started with Hörmander's paper [8, has significantly improved since the works by Folland [7] and by Rothschild and Stein 13. In the latter paper it was shown that every Hörmander operator can be locally approximated by hypoelliptic operators of the following type:

$$
\sum_{j=1}^{m} X_{j}^{2}+Y,
$$

where the $X_{j}$ 's and $Y$ are first-order partial differential operators with polynomial coefficients, left translation invariant and homogeneous of degree one and two, respectively, on a homogeneous Lie group in a real space $\mathbb{R}^{N}$.

As is well known, in the last decades a lot of literature has been devoted to the Hörmander operators on homogeneous Lie groups. We just mention, besides the celebrated papers [8, 4], 7] and [13, the monograph [14], the recent survey article [1, the papers [2], 3] and the references therein. We would also like to explicitly mention the papers [5] and [6] containing a deep analysis of operators with non-smooth coefficients on Lie groups.

The present paper is organized as follows. In Section 1 we introduce the notion of link of groups and in Section 2 we study the algebras of the linked groups. Section 3 and Section 4 are devoted, respectively, to homogeneous and Carnot groups. In Section 3 we show that the link of homogeneous groups is always a homogeneous group. In Section 4 we give sufficient conditions for this to also hold for Carnot groups. Finally in Section 5, by using our general results, we show how to construct sequences of sub-Laplacians and of sub-Kolmogorov operators.

We close the introduction by recalling few basic facts from Lie group theory in $\mathbb{R}^{N}$. A vector field in $\mathbb{R}^{N}$ is a smooth function

$$
X: \mathbb{R}^{N} \longrightarrow \mathbb{R}^{N}, \quad X(x)=\left(a_{1}(x), \ldots, a_{N}(x)\right),
$$

where $a_{j} \in C^{\infty}\left(\mathbb{R}^{N}, \mathbb{R}\right)$ for every $j \in\{1, \ldots, N\}$. 
We identify $X$ with the linear first-order partial differential operator

$$
X:=\sum_{j=1}^{N} a_{j}(x) \partial_{x_{j}}, \quad x=\left(x_{1}, \ldots, x_{N}\right) .
$$

Given a Lie group $\mathbb{G}=\left(\mathbb{R}^{N}, \circ\right)$ we denote by $\mathfrak{g}$ its Lie algebra, i.e. the Lie algebra of the vector fields in $\mathbb{R}^{N}$ which are left translation invariant on $\mathbb{G}$. For every $j \in\{1, \ldots, N\}$, the vector field

$$
X_{j}(x):=\left.\partial_{y_{j}}(x \circ y)\right|_{y=0}, \quad x \in \mathbb{R}^{N},
$$

belongs to $\mathfrak{g}$. The family $\left\{X_{1}, \ldots, X_{N}\right\}$ is a basis of $\mathfrak{g}$ which is called the Jacobian basis of $\mathfrak{g}$.

\section{LINK OF GROUPS}

Let $G_{i}=\left(M_{i}, \circ_{i}\right), i=1,2$, be Lie groups. Assume the manifolds $M_{i}$ can be split as follows:

$$
M_{i}=N_{0} \times N_{i}, \quad i=1,2,
$$

where $N_{0}$ is the underlying manifold of a Lie group $H=\left(N_{0}, \cdot\right)$.

We also assume that the projection

$$
\pi_{i}: N_{0} \times N_{i} \longrightarrow N_{0}, \quad \pi_{i}\left(n_{0}, n_{i}\right)=n_{0},
$$

is a homomorphism of $G_{i}$ on $H, i=1,2$.

Given $\left(n_{0}, n_{i}\right) \in M_{i}, i=1,2$, we define

$$
\left(n_{0}, n_{1}\right) \triangle\left(n_{0}, n_{2}\right):=\left(n_{0}, n_{1}, n_{2}\right) .
$$

Thus, if we set $M=N_{0} \times N_{1} \times N_{2}$, we have

$$
M=M_{1} \triangle M_{2}:=\left\{\left(n_{0}, n_{1}\right) \triangle\left(n_{0}, n_{2}\right) \mid n_{i} \in N_{i}, i=0,1,2\right\} .
$$

In $M$ we introduce the following composition law:

$$
\left(n_{0}, n_{1}, n_{2}\right) \circ\left(n_{0}^{\prime}, n_{1}^{\prime}, n_{2}^{\prime}\right):=\left(\left(n_{0}, n_{1}\right) \circ_{1}\left(n_{0}^{\prime}, n_{1}^{\prime}\right)\right) \triangle\left(\left(n_{0}, n_{2}\right) \circ_{2}\left(n_{0}^{\prime}, n_{2}^{\prime}\right)\right) \text {. }
$$

We explicitly remark that this definition is well posed since

$$
\pi_{i}\left(\left(n_{0}, n_{i}\right) \circ_{i}\left(n_{0}^{\prime}, n_{i}^{\prime}\right)\right)=n_{0} \cdot n_{0}^{\prime} \quad \text { for } i=1,2 .
$$

The following proposition holds.

Proposition 1.1. $G:=(M, \circ)$ is a Lie group.

Proof. We omit the details of the proof, which is completely elementary though lengthy. We just remark that the zero of $M$ is $0=0_{1} \triangle 0_{2}$, where $0_{i}$ denotes the zero of $G_{i}, i=1,2$.

Moreover, if $m \in M, m=m_{1} \triangle m_{2}$, with $m_{i} \in M_{i}$, then

$$
m^{-1}=m_{1}^{-1} \triangle m_{2}^{-1} \text {. }
$$

We shall say that

$$
G=(M, \circ)
$$

is the link of $G_{1}$ and $G_{2}$. 
The following proposition will be useful in what follows.

Proposition 1.2. Let $G_{i}, i=1,2$, be as before. Let

$$
d^{(i)}: G_{i} \longrightarrow G_{i}
$$

be an automorphism of $G_{i}, i=1,2$. Assume

$$
\pi_{1} d^{(1)}=\pi_{2} d^{(2)} .
$$

Then

$$
d:=d^{(1)} \triangle d^{(2)}
$$

is defined as follows:

$$
d\left(n_{0}, n_{1}, n_{2}\right)=\left(d^{(1)}\left(n_{0}, n_{1}\right)\right) \triangle\left(d^{(2)}\left(n_{0}, n_{2}\right)\right)
$$

is an automorphism of $G$.

Proof. The proof is quite easy. We only need to notice that $d$ is well defined thanks to condition (2).

\section{Algebras of linked groups on Real spaces}

Let $\mathbb{R}^{N}, N \geq 3$, be split as follows:

$$
\mathbb{R}^{N}=\mathbb{R}^{p} \times \mathbb{R}^{q} \times \mathbb{R}^{r}
$$

and denote its points by

$$
x=\left(x^{(p)}, x^{(q)}, x^{(r)}\right),
$$

where $x^{(p)} \in \mathbb{R}^{p}, x^{(q)} \in \mathbb{R}^{q}$ and $x^{(r)} \in \mathbb{R}^{r}$. We shall also use the notation

$$
x^{(p, q)}:=\left(x^{(p)}, x^{(q)}\right) \quad \text { and } \quad x^{(p, r)}:=\left(x^{(p)}, x^{(r)}\right) .
$$

Accordingly, for consistency of notation, we shall write $\mathbb{R}^{(p, q)}$ and $\mathbb{R}^{(p, r)}$ instead of $\mathbb{R}^{p} \times \mathbb{R}^{q}$ and $\mathbb{R}^{p} \times \mathbb{R}^{r}$, respectively.

Let

$$
\mathbb{G}_{1}=\left(\mathbb{R}^{(p, q)}, \circ_{1}\right) \quad \text { and } \quad \mathbb{G}_{2}=\left(\mathbb{R}^{(p, r)}, \circ_{2}\right)
$$

be Lie groups. Assume the composition laws in $\mathbb{G}_{1}$ and $\mathbb{G}_{2}$ take the following form:

$$
\begin{aligned}
& x^{(p, q)} \circ_{1} y^{(p, q)}=\left(x^{(p)}, x^{(q)}\right) \circ_{1}\left(y^{(p)}, y^{(q)}\right):=\left(x^{(p)}+y^{(p)}, Q\left(x^{(p, q)}, y^{(p, q)}\right)\right), \\
& x^{(p, r)} \circ_{2} y^{(p, r)}=\left(x^{(p)}, x^{(r)}\right) \circ_{2}\left(y^{(p)}, y^{(r)}\right):=\left(x^{(p)}+y^{(p)}, R\left(x^{(p, r)}, y^{(p, r)}\right)\right)
\end{aligned}
$$

where $Q$ and $R$ are vector functions with values in $\mathbb{R}^{q}$ and $\mathbb{R}^{r}$, respectively.

Then $\mathbb{G}_{1}$ and $\mathbb{G}_{2}$ are linkable and the composition law on $\mathbb{G}:=\mathbb{G}_{1} \triangle \mathbb{G}_{2}$ is given by

$$
x \circ y:=\left(x^{(p)}+y^{(p)}, Q\left(x^{(p, q)}, y^{(p, q)}\right), R\left(x^{(p, r)}, y^{(p, r)}\right)\right) .
$$

We shall denote by $\mathfrak{g}_{i}$ and $\mathfrak{g}$ the Lie algebras of $\mathbb{G}_{i}$ and $\mathbb{G}$ respectively $(i=1,2)$.

Theorem 2.1. Let

$$
\left\{X_{1}, \ldots, X_{p+q}\right\} \quad \text { and } \quad\left\{Y_{1}, \ldots, Y_{p+r}\right\}
$$

be the Jacobian bases of $\mathfrak{g}_{1}$ and $\mathfrak{g}_{2}$ respectively. Then

$$
X_{p+1}, \ldots, X_{p+q} \in \mathfrak{g} .
$$

Moreover, if $R$ is independent of $y^{(p)}$, we also have

$$
X_{1}, \ldots, X_{p} \in \mathfrak{g} .
$$


Analogously,

$$
Y_{p+1}, \ldots, Y_{p+r} \in \mathfrak{g}
$$

and, if $Q$ is independent of $y^{(p)}$, then

$$
Y_{1}, \ldots, Y_{p} \in \mathfrak{g} .
$$

Note. If $1 \leq s \leq N$, we agree to consider any vector field in $\mathbb{R}^{s}$ as a vector field in $\mathbb{R}^{N}$ with the last $N-s$ components equal to zero.

Proof. If $R$ is independent of $y_{j}^{(p, q)}$ for some $j \in\{1, \ldots, p+q\}$, then $X_{j} \in \mathfrak{g}$. Indeed,

$$
\begin{aligned}
\partial_{y_{j}^{(p, q)}}(x \circ y) & =\partial_{y_{j}^{(p, q)}}\left(x^{(p)}+y^{(p)}, Q\left(x^{(p, q)}, y^{(p, q)}\right), R\left(x^{(p, r)}, y^{(p, r)}\right)\right) \\
& =\partial_{y_{j}^{(p, q)}}\left(x^{(p)}+y^{(p)}, Q\left(x^{(p, q)}, y^{(p, q)}\right), 0\right) \\
& =\partial_{y_{j}^{(p, q)}}\left(x^{(p)}+y^{(p)}, Q\left(x^{(p, q)}, y^{(p, q)}\right)\right)=\partial_{y_{j}^{(p, q)}}\left(x^{(p, q)} \circ_{1} y^{(p, q)}\right),
\end{aligned}
$$

so that

$$
\left.\partial_{y_{j}^{(p, q)}}(x \circ y)\right|_{y=0}=\left.\partial_{y_{j}^{(p, q)}}\left(x^{(p, q)} \circ_{1} y^{(p, q)}\right)\right|_{y^{(p, r)}=0^{(p, r)}}=X_{j}
$$

and hence $X_{j} \in \mathfrak{g}$.

Then (5D) and (6) are proved since $R$ is always independent of $y^{(q)}$.

The second part of the theorem can be proved exactly as the first part.

Example 2.2. Let $\mathbb{G}_{1}=\left(\mathbb{R}^{(p, q)}, \circ_{1}\right)$ be a Lie group whose composition law $\circ_{1}$ is as in (31).

Let us now consider a $(p+r) \times(p+r)$ matrix $B$ taking the following block form:

$$
B=\left(\begin{array}{cc}
0 & 0 \\
B_{1} & B_{2}
\end{array}\right)
$$

where $B_{1}$ and $B_{2}$ are, respectively, $r \times p$ and $r \times r$ real matrices. Then the exponential matrix

takes the following block form:

$$
E(t):=\exp (-t B), \quad t \in \mathbb{R},
$$

$$
E(t)=\left(\begin{array}{cc}
I_{p} & 0 \\
E_{1}(t) & E_{2}(t)
\end{array}\right) .
$$

In $\mathbb{R}^{(p, r+1)}$, whose points will be denoted by $\left(x^{(p, r)}, t\right)$, we introduce the composition law

$$
\left(x^{(p, r)}, t\right) \circ_{2}\left(y^{(p, r)}, \tau\right):=\left(y^{(p, r)}+E(\tau) x^{(p, r)}, t+\tau\right) .
$$

It easy to show that

$$
\mathbb{G}_{2}:=\left(\mathbb{R}^{(p, r+1)}, \circ_{2}\right)
$$

is a Lie group.

Moreover, due to (7),

$$
\begin{aligned}
\left(x^{(p, r)}, t\right) \circ_{2}\left(y^{(p, r)}, \tau\right) & =\left(x^{(p)}+y^{(p)}, y^{(r)}+E_{1}(\tau) x^{(p)}+E_{2}(\tau) x^{(r)}, t+\tau\right) \\
& =:\left(x^{(p)}+y^{(p)}, R\left(\left(x^{(p, r)}, t\right),\left(y^{(p, r)}, \tau\right)\right) .\right.
\end{aligned}
$$

It follows that $\mathbb{G}_{1}$ and $\mathbb{G}_{2}$ are linkable and, by Theorem 2.1, since $R$ is independent of $y^{(p)}$, the Jacobian basis of $\mathfrak{g}_{1}$ turns out to be contained in the Jacobian basis of $\mathfrak{g}$ (the Lie algebra of $\mathbb{G}=\mathbb{G}_{1} \triangle \mathbb{G}_{2}$ ).

We now compute the Jacobian basis of $\mathfrak{g}_{2}$. 
From (9) we immediately get

$$
\left.\partial_{y_{j}^{(p, r)}}\left(\left(x^{(p, r)}, t\right) \circ_{2}\left(y^{(p, r)}, \tau\right)\right)\right|_{\left(y^{(p, r)}, \tau\right)=0}=e_{j}, \quad j=1, \ldots, p+r,
$$

where $e_{j}$ denotes the $j$-th element of the canonical basis of $\mathbb{R}^{(p, r+1)}$. Moreover, directly from (8), we obtain

$$
\left.\partial_{\tau}\left(\left(x^{(p, r)}, t\right) \circ_{2}\left(y^{(p, r)}, \tau\right)\right)\right|_{\left(y^{(p, r)}, \tau\right)=0}=\left(\begin{array}{c}
-B x^{(p, r)} \\
1
\end{array}\right) .
$$

Then, the Jacobian basis of $\mathfrak{g}_{2}$ is

$$
\left\{\partial_{x_{j}^{(p, r)}}, j=1, \ldots, p+r\right\} \cup\{Y\},
$$

where

$$
Y:=-\left\langle B x^{(p, r)}, \nabla_{(p, r)}\right\rangle+\partial_{t}
$$

Therefore, by Theorem 2.1, the vector fields

$$
Y \quad \text { and } \quad \partial_{x_{j}^{(p, r)}}, j=p+1, \ldots, p+r,
$$

are left translation invariant on $\mathbb{G}$.

It follows that the Jacobian basis of $\mathfrak{g}$ is given by

$$
X_{1}, \ldots, X_{p+q}, \partial_{x_{p+1}^{(p, r)}}, \ldots, \partial_{x_{p+r}^{(p, r)}}, Y
$$

where we have denoted by $\left\{X_{1}, \ldots, X_{p+q}\right\}$ the Jacobian basis of $\mathfrak{g}_{1}$.

\section{LINK OF HOMOGENEOUS GROUPS}

We shall call group of dilations in $\mathbb{R}^{N}$ any family $\left(d_{\lambda}\right)_{\lambda>0}$ of diagonal linear map of the form

$$
d_{\lambda}: \mathbb{R}^{N} \longrightarrow \mathbb{R}^{N}, d_{\lambda}\left(x_{1}, \ldots, x_{N}\right)=\left(\lambda^{\alpha_{1}} x_{1}, \ldots, \lambda^{\alpha_{N}} x_{N}\right),
$$

where the $\alpha_{j}$ 's are suitable real positive numbers.

A Lie group $\left(\mathbb{R}^{N}, \circ\right)$ will be called a homogeneous Lie group if there exists a group of dilations $\left(d_{\lambda}\right)_{\lambda>0}$ such that $d_{\lambda}$ is a homomorphism of $\left(\mathbb{R}^{N}, \circ\right)$ for every $\lambda>0$.

We shall denote by

$$
\mathbb{G}=\left(\mathbb{R}^{N}, \circ, d_{\lambda}\right)
$$

a homogeneous Lie group endowed with a group of dilations given by $\left(d_{\lambda}\right)_{\lambda>0}$. Let

$$
\mathbb{G}_{1}=\left(\mathbb{R}^{(p, q)}, \circ_{1}, d_{\lambda}^{(1)}\right) \quad \text { and } \quad \mathbb{G}_{2}=\left(\mathbb{R}^{(p, r)}, \circ_{2}, d_{\lambda}^{(2)}\right)
$$

be homogeneous Lie groups and suppose the composition laws $\circ_{1}$ and $\circ_{2}$ are defined as in (3) and (4). We also assume that the dilations $d_{\lambda}^{(1)}$ and $d_{\lambda}^{(2)}$ take the following form:

$$
\begin{aligned}
& d_{\lambda}^{(1)}\left(x^{(p)}, x^{(q)}\right)=\left(\lambda x^{(p)}, \rho_{\lambda}^{(1)}\left(x^{(q)}\right)\right), \\
& d_{\lambda}^{(2)}\left(x^{(p)}, x^{(r)}\right)=\left(\lambda x^{(p)}, \rho_{\lambda}^{(2)}\left(x^{(r)}\right)\right) .
\end{aligned}
$$

These assumptions imply that $\mathbb{G}_{1}$ and $\mathbb{G}_{2}$ are linkable (in the sense of the previous section) and that the dilations $d_{\lambda}^{(1)}$ and $d_{\lambda}^{(2)}$ satisfy the hypotheses of Proposition 1.2, Then

$$
\mathbb{G}=\mathbb{G}_{1} \triangle \mathbb{G}_{2}
$$


is a homogeneous Lie group with respect to the dilations

$$
d_{\lambda}: \mathbb{R}^{N} \longrightarrow \mathbb{R}^{N}, \quad d_{\lambda}\left(x^{(p)}, x^{(q)}, x^{(r)}\right)=\left(\lambda x^{(p)}, \rho_{\lambda}^{(1)}\left(x^{(q)}\right), \rho_{\lambda}^{(2)}\left(x^{(r)}\right)\right) .
$$

In what follows, we shall call

$$
\mathbb{G}=\left(\mathbb{R}^{N}, \circ, d_{\lambda}\right)
$$

the link of the homogeneous groups $\mathbb{G}_{1}$ and $\mathbb{G}_{2}$.

Example 3.1. Let $\mathbb{G}_{1}=\left(\mathbb{R}^{(p, q)}, \circ_{1}, d_{\lambda}^{1}\right)$ be a homogeneous Lie group. Assume the composition law $\circ_{1}$ and the dilation $d_{\lambda}^{1}$ as in (3) and (11), respectively.

Let us now consider a Kolmogorov-type group, i.e. a group

$$
\mathbb{K}=\left(\mathbb{R}^{(p, r+1)}, \circ_{2}, d_{\lambda}^{(2)}\right)
$$

where $\mathrm{O}_{2}$ is the composition law (8) related to the following $(p+r) \times(p+r)$ matrix $B$ :

$$
B=\left(\begin{array}{ccccc}
0 & 0 & 0 & \ldots & 0 \\
B_{1} & 0 & 0 & \ldots & 0 \\
0 & B_{2} & \ldots & \ldots & \ldots \\
\vdots & \vdots & \ddots & \vdots & \vdots \\
0 & 0 & 0 & B_{k} & 0
\end{array}\right)
$$

Assume that $B_{j}$ is a maximum rank matrix of dimension $r_{j} \times r_{j-1}$, with $r_{0}=p \geq$ $r_{1} \geq \ldots \geq r_{k} \geq 1$ and $r_{0}+r_{1}+\ldots+r_{k}=N$.

The dilations

$$
\begin{gathered}
d_{\lambda}^{(2)}: \mathbb{R}^{(p, r+1)} \longrightarrow \mathbb{R}^{(p, r+1)}, \\
d_{\lambda}^{(2)}\left(x^{(p)}, x^{\left(r_{1}\right)}, \ldots, x^{\left(r_{k}\right)}, t\right)=\left(\lambda x^{(p)}, \lambda^{3} x^{\left(r_{1}\right)}, \ldots, \lambda^{2 k+1} x^{\left(r_{k}\right)}, \lambda^{2} t\right), \quad \lambda>0,
\end{gathered}
$$

make $\mathbb{K}$ a homogeneous Lie group (see [12, where the Kolmogorov-type groups were first introduced).

Then, $\mathbb{G}_{1}$ and $\mathbb{K}$ are linkable homogeneous groups.

\section{Link of Carnot groups}

Let $\mathbb{G}=\left(\mathbb{R}^{N}, \circ, d_{\lambda}\right)$ be a homogeneous group and assume that

$$
d_{\lambda}\left(x_{1}, \ldots, x_{N}\right)=\left(\lambda^{\alpha_{1}} x_{1}, \ldots, \lambda^{\alpha_{N}} x_{N}\right),
$$

where $\alpha_{j} \in \mathbb{N}$ for every $j \in\{1, \ldots, N\}$. Let us denote by $\Lambda_{\mathbb{G}}$ the subset of the indices $j \in\{1, \ldots, N\}$ such that $\alpha_{j}=1$, and assume that the cardinality of $\Lambda_{\mathbb{G}}$ is greater than or equal to 2 .

We say that $\mathbb{G}$ is a Carnot group if the Lie algebra $\mathfrak{g}$ of $\mathbb{G}$ is generated by the left translation invariant vector fields

$$
X_{j}, \quad j \in \Lambda_{\mathbb{G}},
$$

such that $X_{j}(0)=\partial_{x_{j}}$. These vector fields are called the generators of $\mathbb{G}$. It can be proved that, if $j \in \Lambda_{\mathbb{G}}$, the $j$-th component of the composition law is Euclidean, i.e.

$$
(x \circ y)_{j}=x_{j}+y_{j} \quad \forall j \in \Lambda_{\mathbb{G}} .
$$

We want to show that, under suitable conditions, the link of two Carnot groups is a Carnot group.

So let

$$
\mathbb{G}_{1}=\left(\mathbb{R}^{(p, q)}, \circ_{1}, d_{\lambda}^{(1)}\right) \quad \text { and } \quad \mathbb{G}_{2}=\left(\mathbb{R}^{(p, r)}, \circ_{2}, d_{\lambda}^{(2)}\right)
$$


be Carnot groups. Up to a rearrangement of the variables we may and do assume

$$
\Lambda_{\mathbb{G}_{1}}=\{1, \ldots, p\}, \quad \Lambda_{\mathbb{G}_{2}}=\{1, \ldots, m\}, \quad \text { with } \quad p \leq m \leq p+r .
$$

As a consequence, $d_{\lambda}^{(1)}$ and $d_{\lambda}^{(2)}$ take the form

$$
\begin{gathered}
d_{\lambda}^{(1)}\left(x^{(p, q)}\right)=\left(\lambda x^{(p)}, \rho_{\lambda}^{(1)}\left(x^{(q)}\right)\right), \\
d_{\lambda}^{(2)}\left(x^{(p, r)}\right)=\left(\lambda x^{(p)}, \rho_{\lambda}^{(2)}\left(x^{(r)}\right)\right)=\left(\lambda x^{(p)}, \lambda x^{(m-p)}, \sigma_{\lambda}^{(2)}\left(x^{(p+r-m)}\right)\right) .
\end{gathered}
$$

Then, $\mathbb{G}_{1}$ and $\mathbb{G}_{2}$ are linkable homogeneous Lie groups with dilations given by:

$$
\begin{aligned}
d_{\lambda}\left(x^{(p)}, x^{(q)}, x^{(r)}\right) & =d_{\lambda}\left(x^{(p)}, x^{(q)}, x^{(m-p)}, x^{(p+r-m)}\right) \\
& =\left(\lambda x^{(p)}, \rho_{\lambda}^{(1)}\left(x^{(q)}\right), \lambda x^{(m-p)}, \sigma_{\lambda}^{(2)}\left(x^{(p+r-m)}\right)\right) .
\end{aligned}
$$

However, in general, $\mathbb{G}:=\mathbb{G}_{1} \triangle \mathbb{G}_{2}$ is not a Carnot group.

In order to give a condition ensuring that $\mathbb{G}$ is a Carnot group, we first remark that the composition law $\circ_{1}$ can be written as follows:

$$
x^{(p, q)} \circ_{1} y^{(p, q)}:=\left(x^{(p)}+y^{(p)}, R\left(x^{(p, q)}, y^{(p, q)}\right)\right) .
$$

Theorem 4.1. Assume the following conditions are satisfied:

(H1) $R$ is independent of $y^{(p)}$.

$(\mathrm{H} 2) \partial_{x_{j}^{(p)}}$ is left translation invariant on $\mathbb{G}_{2}$ for $1 \leq j \leq p$.

Then

$$
\mathbb{G}=\mathbb{G}_{1} \triangle \mathbb{G}_{2}
$$

is a Carnot group with $m$ generators.

More precisely, if $p<m, X_{1}, \ldots, X_{p}$ are the generators of $\mathbb{G}_{1}$ and $\partial_{x_{1}^{(p)}}, \ldots$, $\partial_{x_{p}^{(p)}}, Y_{p+1}, \ldots, Y_{m}$ are the generators of $\mathbb{G}_{2}$, then the vector fields

$$
X_{1}, \ldots, X_{p}, Y_{p+1}, \ldots, Y_{m}
$$

are the generators of $\mathbb{G}$.

The proof of this theorem will straightforwardly follow from the next lemma and corollary.

Lemma 4.2. Let $Y$ be a vector field in $\mathbb{R}^{(p, q, r)}$ taking the form

$$
Y=\sum_{k=1}^{r} b^{(k)} \partial_{x_{k}^{(r)}}
$$

with $b^{(k)}$ independent of $x^{(q)}, k=1, \ldots, r$.

Then

$$
\left[X_{j}, Y\right]=\left[\partial_{x_{j}^{(p)}}, Y\right] \quad \forall j \in\{1, \ldots, p\} .
$$

Proof. From the general theory of homogeneous Lie groups on Euclidean spaces it follows that $X_{j}, j \in\{1, \ldots, p\}$, can be written as

$$
X_{j}=\partial_{x_{j}^{(p)}}+\widetilde{X}_{j},
$$

where

$$
\widetilde{X}_{j}=\sum_{k=1}^{q} a_{j}^{(k)} \partial_{x_{k}^{(q)}}, \quad a_{j}^{(k)}=a_{j}^{(k)}\left(x^{(p, q)}\right) .
$$

Then $\left[\widetilde{X}_{j}, Y\right]=0$ and the assertion follows. 
Corollary 4.3. Let

$$
\partial_{x_{1}^{(p)}}, \ldots, \partial_{x_{p}^{(p)}}, Y_{p+1}, \ldots, Y_{m}, \ldots, Y_{p+r}
$$

be the Jacobian basis of $\mathfrak{g}_{2}$. Then

$$
Y_{p+i} \in \operatorname{Lie}\left\{X_{1}, \ldots, X_{p}, Y_{p+1}, \ldots, Y_{m}\right\} \quad \forall i \in\{1, \ldots, r\} .
$$

Proof. From the general theory of homogeneous Lie groups on Euclidean spaces, it follows that each vector field

$$
Y_{p+1}, \ldots, Y_{m}, \ldots, Y_{p+r}
$$

can be written as in (14) with coefficients independent of $x^{(q)}$.

Therefore the result follows by applying Lemma 4.2

Proof of Theorem 4.1. Let us denote by

$$
X_{1}, \ldots, X_{p}, X_{p+1}, \ldots, X_{p+q}
$$

the Jacobian basis of $\mathfrak{g}_{1}$. Moreover, let (15) be the Jacobian basis of $\mathfrak{g}_{2}$. Hypothesis (H1) and Theorem 2.1 imply that

$$
X_{1}, \ldots, X_{p}, X_{p+1}, \ldots, X_{p+q}, Y_{p+1}, \ldots, Y_{m}, \ldots, Y_{p+r} \in \mathfrak{g} .
$$

On the other hand,

$$
\begin{aligned}
X_{j}(0)=\partial_{x_{j}^{(p)}} & \text { if } \quad j \in\{1, \ldots, p\}, \\
X_{p+j}(0)=\partial_{x_{j}^{(q)}} & \text { if } \quad j \in\{1, \ldots, q\}, \\
Y_{p+i}(0)=\partial_{x_{i}^{(r)}} & \text { if } \quad i \in\{1, \ldots, r\} .
\end{aligned}
$$

Then, the vector fields in (16) are linearly independent since they are linearly independent at the origin.

Since $\operatorname{dim} \mathfrak{g}=\operatorname{dim}\left(\mathbb{R}^{(p, q)} \triangle \mathbb{R}^{(p, r)}\right)=p+q+r$, we get that (16) is the Jacobian basis of $\mathfrak{g}$. The structure of the dilations in (13) implies that

$$
\Lambda_{\mathbb{G}}=\{1, \ldots, p\} \cup\{p+q+1, \ldots, q+m\} .
$$

Hence the cardinality of $\Lambda_{\mathbb{G}}$ is equal to $m$.

In order to complete the proof, we have to show that

$$
\operatorname{dim}\left(\operatorname{Lie}\left\{X_{1}, \ldots, X_{p}, Y_{p+1}, \ldots, Y_{m}\right\}\right)=p+q+r .
$$

It is quite obvious that

$$
X_{j} \in \operatorname{Lie}\left\{X_{1}, \ldots, X_{p}, Y_{1}, \ldots, Y_{m}\right\} \quad \forall j \in\{1, \ldots, p+q\} .
$$

Moreover, by Corollary 4.3 .

$$
Y_{p+i} \in \operatorname{Lie}\left\{X_{1}, \ldots, X_{p}, Y_{1}, \ldots, Y_{m}\right\} \quad \forall i \in\{1, \ldots, r\} .
$$

Then (17) follows from the first part of the proof.

Example 4.4. Let $\mathbb{G}_{1}=\left(\mathbb{R}^{(p, q)}, \circ_{1}, d_{\lambda}^{(1)}\right)$ be a Carnot group with $p$ generators. Assume $\Lambda_{\mathbb{G}_{1}}=\{1, \ldots, p\}$ and denote by

$$
X_{1}, \ldots, X_{p}
$$

the generators of $\mathbb{G}_{1}$.

Let us now consider a Carnot-Kolmogorov type group on $\mathbb{R}^{(p, r+1)}$, i.e. a homogeneous Lie group

$$
\mathbb{C K}:=\left(\mathbb{R}^{(p, r+1)}, \circ_{2}, d_{\lambda}^{(2)}\right)
$$


whose composition law is defined as in (8) (see Example 2.2) and the matrix $B$ is given by (12). Moreover the dilation $d_{\lambda}^{(2)}$ is defined as follows:

$$
\begin{aligned}
d_{\lambda}^{(2)}\left(x^{(p, r+1)}\right) & =d_{\lambda}^{(2)}\left(x^{(p)}, x^{(r+1)}\right) \\
& =d_{\lambda}^{(2)}\left(x^{(p)}, x^{\left(r_{1}\right)}, \ldots, x^{\left(r_{k}\right)}, t\right) \\
& =\left(\lambda x^{(p)}, \lambda^{2} x^{\left(r_{1}\right)}, \ldots, \lambda^{k+1} x^{\left(r_{k}\right)}, \lambda t\right) .
\end{aligned}
$$

Then, $\mathbb{C K}$ is a Carnot group with $p+1$ generators and

$$
\Lambda_{\mathbb{C K}}=\{1, \ldots, p\} \cup\{p+r+1\}
$$

(see also [2]). The generators of $\mathbb{C K}$ are the vector fields

$$
\partial_{x_{1}^{(p)}}, \ldots, \partial_{x_{p}^{(p)}}, Y
$$

where

$$
Y=-\left\langle B x^{(p, r)}, D_{(p, r)}\right\rangle+\partial_{t} ;
$$

see (10). Here $\langle\cdot, \cdot\rangle$ and $D_{(p, r)}$ denote the inner product and the Euclidean gradient in $\mathbb{R}^{(p, r)}$, respectively.

Then, since the function $R$ in (9) is independent of $y^{(p)}, \mathbb{G}_{1}$ and $\mathbb{C K}$ are linkable homogeneous Lie groups and the linked group

$$
\mathbb{G}:=\mathbb{G}_{1} \triangle \mathbb{C K}
$$

is a Carnot group whose generators are

$$
X_{1}, \ldots, X_{p}, Y
$$

(see Theorem 4.1).

Definition 4.5. We shall call the vector field $Y$ in (18) a derivative operator transverse to $\mathbb{G}_{1}$.

\section{An APplication}

5.1. Sequences of sub-Laplacians. Let $\mathbb{G}_{1}$ be a Carnot group with generators

$$
X_{1}, \ldots, X_{p} \text {. }
$$

Then

$$
\Delta_{\mathbb{G}}:=\sum_{j=1}^{p} X_{j}^{2}
$$

is a second-order partial differential operator which is called the canonical subLaplacian on $\mathbb{G}_{1}$. Since

$$
\mathfrak{g}_{1}:=\operatorname{Lie}\left\{X_{1}, \ldots, X_{p}\right\},
$$

$\mathfrak{g}_{1}$ being the Lie algebra of $\mathbb{G}_{1}, \Delta_{\mathbb{G}}$ is hypoelliptic by the well-known theorem of Hörmander [8].

Let us now consider a derivative operator $Y_{1}$ transverse to $\mathbb{G}_{1}$ and denote by $\mathbb{C K}_{1}$ the Carnot-Kolmogorov type group related to $Y_{1}$ (see Example 4.4).

Then

$$
X_{1}, \ldots, X_{p}, Y_{1}
$$

are the generators of the Carnot group

$$
\mathbb{G}_{2}:=\mathbb{G}_{1} \triangle \mathbb{C K}_{1} .
$$


Hence

$$
\begin{aligned}
\Delta_{\mathbb{G}_{2}} & =\sum_{j=1}^{p} X_{j}^{2}+Y_{1}^{2} \\
& =\Delta_{\mathbb{G}_{1}}+Y_{1}^{2} .
\end{aligned}
$$

We can continue this procedure by choosing a derivative operator $Y_{2}$ transverse to $\mathbb{G}_{2}$. Thus, denoting by $\mathbb{C K}_{2}$ the Carnot-Kolmogorov type group related to $Y_{2}$, we have that

$$
\Delta_{\mathbb{G}_{3}}=\Delta_{\mathbb{G}_{2}}+Y_{2}^{2}=\Delta_{\mathbb{G}_{1}}+Y_{1}^{2}+Y_{2}^{2}
$$

is the canonical sub-Laplacian on the Carnot group

$$
\mathbb{G}_{3}:=\mathbb{G}_{2} \triangle \mathbb{C K}_{2} \equiv\left(\mathbb{G}_{1} \triangle \mathbb{C K}_{1}\right) \triangle \mathbb{C K}_{2} .
$$

By iterating this argument, we can construct a sequence of Carnot groups $\left(\mathbb{G}_{n}\right)_{n \geq 1}$ such that

$$
\mathbb{G}_{n+1}:=\mathbb{G}_{n} \triangle \mathbb{C K}_{n},
$$

$\mathbb{C K}_{n}$ being a Carnot-Kolmogorov type group related to a derivative operator $Y_{n}$ transverse to $\mathbb{G}_{n}$.

We also have

$$
\begin{aligned}
\Delta_{\mathbb{G}_{n+1}} & =\Delta_{\mathbb{G}_{n}}+Y_{n}^{2} \\
& =\Delta_{\mathbb{G}_{1}}+\sum_{k=1}^{n} Y_{k}^{2} .
\end{aligned}
$$

5.2. Sequences of sub-Kolmogorov operators. Let $\mathbb{G}_{1}$ be a Carnot group with generators $X_{1}, \ldots, X_{p}$. Let $Y_{1}$ be a derivative operator transverse to $\mathbb{G}_{1}$. Then we call

$$
\mathcal{K}_{1}:=\Delta_{\mathbb{G}_{1}}+Y_{1}=\sum_{j=1}^{p} X_{j}^{2}+Y_{1}
$$

a sub-Kolmogorov operator (see 9] where this notion was first introduced).

Then, $\mathcal{K}_{1}$ is left translation invariant on the homogeneous linked group

$$
\mathbb{K}_{1}=\mathbb{G}_{1} \triangle \mathbb{K}_{1}
$$

where $\mathbb{K}_{1}$ is the Kolmogorov-type group related to $Y_{1}$ (see Example 3.1). We also know that $\mathcal{K}_{1}$ is homogeneous of degree two with respect to the dilations of $\mathbb{K}_{2}$. Moreover, the composition law on $\mathbb{K}_{2}$ is the same composition law on $\mathbb{G}_{2}$ of the previous example. Then $\mathbb{K}_{2}$ and $\mathbb{G}_{2}$ have the same Lie algebra, which is $\operatorname{Lie}\left\{X_{1}, \ldots, X_{p}, Y_{1}\right\}$. It follows that $\mathcal{K}_{2}$ is hypoelliptic, since it satisfies the Hörmander condition. Now, let us now consider the sequence of sub-Laplacians $\left(\Delta_{\mathbb{G}_{n}}\right)_{n \geq 1}$ constructed in the previous section, and let $\left(Y_{n}\right)_{n \geq 1}$ be the related sequence of transverse derivative operators, i.e. $Y_{n}$ is transverse to $\mathbb{G}_{n}$. Then

$$
\mathcal{K}_{n}=\Delta_{\mathbb{G}_{n}}+Y_{n}
$$

is a sub-Kolmogorov operator for every $n \in \mathbb{N}$.

We would like to explicitly remark that

$$
\mathcal{K}_{n+1}=\Delta_{\mathbb{G}_{1}}+\left(Y_{1}^{2}+\ldots+Y_{n}^{2}\right)+Y_{n+1}, \quad n \geq 1 .
$$

All these operators are contained in the classes studied in [9] and [10]. 


\section{REFERENCES}

[1] G.K. Alexopoulos, Sub-Laplacians with drift on Lie groups of polynomial volume growth, Mem. Amer. Math. Soc. 155 (2002), no. 739. MR.1878341 (2003c:22015)

[2] A. Bonfiglioli, E. Lanconelli and F. Uguzzoni, Uniform Gaussian estimates for the fundamental solutions for heat operators on Carnot groups, Advances Diff. Equat. 7 (2002),1153-1192. MR1919700 (2003f:35054)

[3] A. Bonfiglioli and F. Uguzzoni, Families of diffeomorphic sub-Laplacians and free Carnot groups, Forum Math., 16 (2004), 403-415. MR.2050190(2005f:22029)

[4] J.M. Bony, Principe du maximum, inégalité de Harnack et unicité du problème de Cauchy pour les opérateurs elliptiques dégénérés, Ann. Inst. Fourier, Grenoble 19 (1969), 277-304. MR0262881 (41:7486)

[5] M. Bramanti and L. Brandolini, $L^{p}$ estimates for nonvariational hypoelliptic operators with VMO coefficients, Trans. Amer. Math. Soc. , 352 (2000), no. 2, 781-822. MR.1608289 (2000c:35026)

[6] M. Bramanti and L. Brandolini, $L^{p}$ estimates for uniformly hypoelliptic operators with discontinuous coefficients on homogeneous groups, Rend. Sem. Mat. Univ. Politec. Torino, 58 (2000), no. 4, 389-493. MR.1962808 (2004c:35058)

[7] G.B. Folland, Subelliptic estimates and function spaces on nilpotent Lie groups, Ark. Mat. 13 (1975), 161-207. MR0494315 (58:13215)

[8] L. Hörmander, Hypoelliptic second-order differential equations, Acta Math. 119 (1967), 147171. MR0222474 (36:5526)

[9] A.E. Kogoj and E. Lanconelli, An invariant Harnack inequality for a class of hypoelliptic ultraparabolic equations, Mediterr. J. Math. 1 (2004), 51-80. MR2088032 (2006b:35042)

[10] A.E. Kogoj and E. Lanconelli, One-Side Liouville Theorems for a Class of Hypoelliptic Ultraparabolic Equations, Contemporary Math. 368 (2005), 305-312. MR2126477 (2005j:35025)

[11] E. Lanconelli, A. Pascucci and S. Polidoro, Linear and nonlinear ultraparabolic equations of Kolmogorov type arising in diffusion theory and finance, in the book "Nonlinear Problems in Mathematical Physics and Related Topics, II" in Honor of Professor O.A. Ladyzhenskaya, Int. Math. Series 2 (2002), 243-265. MR1972000 (2004c:35238)

[12] E. Lanconelli and S. Polidoro, On a class of hypoelliptic evolution operators, Rend. Sem. Mat. Univ. Pol. Torino 52 (1994), Partial Diff. Eqs., 29-63. MR1289901 (95h:35044)

[13] L.P. Rothschild and E.M. Stein, Hypoelliptic differential operators and nilpotent groups, Acta Math. 137 (1976), 247-320. MR0436223 (55:9171)

[14] N.T. Varopoulos, L. Saloff-Coste and T. Coulhon, Analysis and geometry on groups, Cambridge Tracts in Mathematics 100, Cambridge University Press (1992), Cambridge. MR.1218884 (95f:43008)

Dipartimento di Matematica, Università di Bologna, Piazza di Porta San Donato, 5, IT-40126 BolOGNA, ITALY

E-mail address: kogoj@dm.unibo.it

Dipartimento di Matematica, Università di Bologna, Piazza di Porta San Donato, 5, IT-40126 Bologna, ITALY

E-mail address: lanconel@dm.unibo.it 